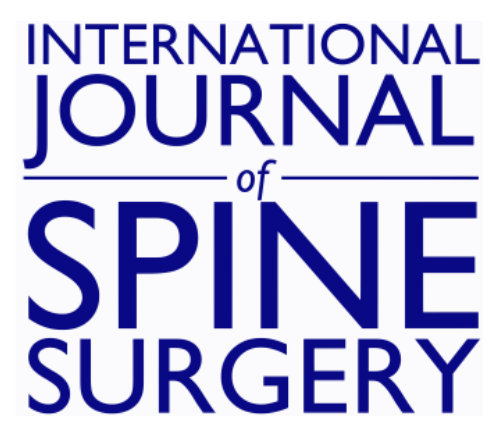

\title{
Patient Radiation Exposure Associated With the Use of Computer Navigation During Spinal Fusion
}

\author{
ALAN W. REYNOLDS, FRANCES HITE PHILP, SACHIN GANDHI and GARY L. SCHMIDT
}

Int J Spine Surg 2020, 14 (4) 534-537

doi: https://doi.org/10.14444/7070

http://ijssurgery.com/content/14/4/534

This information is current as of April 25, 2023.

Email Alerts Receive free email-alerts when new articles cite this article. Sign up at:

http://ijssurgery.com/alerts 


\title{
Patient Radiation Exposure Associated With the Use of Computer Navigation During Spinal Fusion
}

\author{
ALAN W. REYNOLDS, MD, FRANCES HITE PHILP, MS, SACHIN GANDHI, MD, \\ GARY L. SCHMIDT, MD, MBA \\ Department of Orthopaedic Surgery, Allegheny General Hospital, Pittsburgh, Pennsylvania
}

\begin{abstract}
Background: Calibration of computer navigation for spinal fusion is most commonly conducted using either a preoperative computed tomography $(\mathrm{CT})$ scan or intraoperative O-arm scanning. This study aimed to directly compare patient radiation exposure from intraoperative O-arm use for pedicle screw placement versus typical diagnostic lumbar spine CT studies.

Methods: A retrospective review of patients undergoing O-arm navigated lumbar spine fusion procedures was performed to record radiation exposure as the primary outcome, as well as surgical and demographic details. The same was done for a control group of patients undergoing lumbar spine CT scans.

Results: A total of 83 patients undergoing lumbar spine fusion with O-arm navigation were included, as well as 105 unique patients who underwent a lumbar spine CT. The 2 groups were similar in terms of average age ( 60.2 versus $60.5, P=.90)$, average height (170 $\mathrm{cm}$ versus $169 \mathrm{~cm}, P=.50)$, and average weight (92.6 kg versus $90.9 \mathrm{~kg}, P=.62)$. Dose-length product for O-arm navigated procedures was $798.3 \mathrm{mGy}-\mathrm{cm}$ and $924.2 \mathrm{mGy}-\mathrm{cm}$ for CT scans $(P=.064)$. Subgroup analysis revealed 18 patients who had both an O-arm navigated surgery and a lumbar spine CT. In this group the average dose-length product for O-arm surgeries was $806.2 \mathrm{mGy}-\mathrm{cm}$ and $822.1 \mathrm{mGy}-\mathrm{cm}$ for CT scans $(P=$ .92)

Conclusion: This study revealed no statistically or clinically significant differences between patient radiation exposure for O-arm operative navigation compared to lumbar spine CT.

Clinical Relevance: Given the similarity in radiation exposure, surgeons should rely on other factors to guide decision making in regard to mode of imaging for navigation. Knowledge of this comparison and total radiation exposure will also be useful for patient education and shared decision making in regard to navigated procedures.
\end{abstract}

Lumbar Spine

Keywords: lumbar fusion, navigation, radiation, spine, spine surgery, robotic

\section{INTRODUCTION}

Computer navigation has been increasingly adopted for hardware placement during spinal fusion. ${ }^{1}$ Multiple studies have documented the high accuracy and safety of pedicle screw placement achieved when taking advantage of this technology. ${ }^{2,3}$

Calibration of computer navigation for spinal fusion is most commonly conducted using either a preoperative computed tomography (CT) scan or intraoperative O-arm scanning. A patient's preoperative CT scan is loaded into the navigation system and then "point match" registering is done during surgery whereby navigated tools are touched to known intraoperative anatomic landmarks on the patient. These landmarks are then matched to the same points on the CT scan.
Alternatively, an intraoperative $\mathrm{O}$-arm scan is performed and navigated tools are referenced from a fixed clamp in this scan to determine the spatial location of the tools.

Radiation doses delivered to the patient have generally been found to be higher in cases using $\mathrm{O}$ arm scanning for pedicle screw placement, compared to cases using fluoroscopy. ${ }^{4-6}$ However, several studies have reported $\mathrm{O}$-arm radiation doses to be comparable or slightly less than doses associated with an average diagnostic CT scan. ${ }^{7-11}$ More evidence is needed to define variations, if any exist, in the amount of radiation incurred via each of these modalities used in spinal fusion procedures. To this end, the goal of this retrospective study was to compare cumulative radiation exposure in patients undergoing spinal fusion utilizing $\mathrm{O}$-arm navigation versus a typical lumbar CT scan. 


\section{METHODS}

\section{Eligible Population}

This study was performed as a retrospective casecontrol investigation. All adult patients undergoing a lumbar spine fusion using O-arm navigation by a single, fellowship-trained, orthopedic spine surgeon from calendar years 2015 to 2018 were initially included in the study. Only patients with incomplete operative or radiology records were excluded. A control group consisting of 105 randomly selected patients undergoing a regular lumbar spine CT scan was also identified by review of radiology records.

\section{Outcome Measures}

The primary outcome measure was radiation dose as measured in dose-length product (DLP) for both $\mathrm{O}$-arm navigated procedures and CT scans. Demographic and surgical details were also collected including age, sex, weight, body mass index (BMI), number of levels fused, and length of surgery.

\section{Data Collection}

All data were collected from the electronic medical record, including patient charts, operative reports, and radiology procedure details. Prior to data collection being performed, this study was reviewed and approved by the Institutional Review Board.

\section{Statistical Methods}

Data are expressed as mean and SD of the mean. For comparison of continuous variables, Student $t$ tests were used. A $P$ value of $\leq .05$ was considered statistically significant.

\section{RESULTS}

A total of 83 patients undergoing lumbar spine fusion with $\mathrm{O}$-arm navigation were included, as well as 105 unique patients who underwent a lumbar spine CT. The 2 groups were similar in terms of average age $(60.2$ versus $60.5, P=.90)$, average height $(170 \mathrm{~cm}$ versus $170 \mathrm{~cm}, P=.50)$, and average weight (92.6 $\mathrm{kg}$ versus $90.5 \mathrm{~kg}, P=.62$ ). Average DLP for O-arm navigated procedures was 798.3 $\mathrm{mGy}-\mathrm{cm}$ and $924.2 \mathrm{mGy}-\mathrm{cm}$ for CT scans $(P=$ .064). A summary of these data are presented in Table 1.
Table 1. Demographic information and radiation exposure for patients undergoing $\mathrm{O}$-arm navigated lumbar spine fusion versus patients with lumbar computed tomography (CT) scan.

\begin{tabular}{lccc}
\hline & $\begin{array}{c}\text { O-Arm Group, } \\
\text { mean } \pm \mathbf{S D}, \\
\mathbf{n}=\mathbf{8 3}\end{array}$ & $\begin{array}{c}\text { Lumbar CT Group, } \\
\text { mean } \pm \mathbf{S D}, \\
\mathbf{n}=\mathbf{1 0 5}\end{array}$ & $\begin{array}{c}\boldsymbol{P} \text { value } \\
(\boldsymbol{t} \text { test })\end{array}$ \\
\hline Age & $60.2 \pm 12.8$ & $60.5 \pm 17.5$ & .90 \\
Height $(\mathrm{cm})$ & $170.0 \pm 10$ & $169.0 \pm 10$ & .50 \\
Weight $(\mathrm{kg})$ & $92.6 \pm 21.4$ & $90.9 \pm 24.4$ & .62 \\
BMI & $32.0 \pm 6.8$ & $31.5 \pm 7.7$ & .64 \\
DLP (mGy-cm) & $798.3 \pm 336.5$ & $924.2 \pm 536.7$ & .064 \\
\hline
\end{tabular}

Abbreviations: BMI, body mass index; DLP, dose-length product; mGy-cm, milligray-centimeter.

Subgroup analysis revealed 18 patients who had both an $\mathrm{O}$-arm navigated surgery and a lumbar spine CT. In this group the average DLP for O-arm surgeries was $806.2 \mathrm{mGy}-\mathrm{cm}$ and $822.1 \mathrm{mGy}-\mathrm{cm}$ for CT scans $(P=.92)$, as shown in Table 2 .

\section{DISCUSSION}

This study was successful in comparing patient radiation exposure for $\mathrm{O}$-arm navigated lumbar spine fusions and lumbar spine CT scans in 2 groups of subjects similar in age and body habitus. Our analysis found no statistically significant difference in radiation exposure between these 2 groups. It is notable, however, that the mean lumbar CT group exposure was higher and these results trended towards statistical significance for $\mathrm{O}$-arm navigation resulting in less patient radiation exposure $(P=$ $.064)$.

A strength of this study is the subgroup analysis that was possible for 18 patients who had both an $\mathrm{O}$-arm navigated procedure and lumbar spine CT, and thus were able to serve as their own controls. In this group there was again no statistically significant difference in radiation exposure. There was again a lower exposure for the $\mathrm{O}$-arm but the difference was of lesser magnitude than in the complete study group.

Table 2. Subgroup of patients who underwent both an O-arm navigated lumbar spine fusion and a lumbar spine computed tomography (CT) scan.

\begin{tabular}{lccc}
\hline & $\begin{array}{c}\text { O-Arm Subgroup, } \\
\text { mean } \pm \mathbf{S D}, \\
\mathbf{n}=\mathbf{1 8}\end{array}$ & $\begin{array}{c}\text { Lumbar CT } \\
\text { Subgroup, } \\
\text { mean } \pm \mathbf{S D}, \\
\mathbf{n}=\mathbf{1 8}\end{array}$ & $\begin{array}{c}\boldsymbol{P} \text { value } \\
(\boldsymbol{t} \text { test })\end{array}$ \\
\hline Age & $\mathbf{5 9 . 6} \pm \mathbf{1 6 . 1}$ & Same population & NA \\
Height $(\mathrm{cm})$ & $\mathbf{1 7 0} \pm \mathbf{1 0 . 0}$ & Same population & NA \\
Weight $(\mathrm{kg})$ & $\mathbf{9 4 . 4} \pm \mathbf{1 9 . 2}$ & Same population & NA \\
BMI & $\mathbf{3 1 . 5} \pm \mathbf{7 . 8}$ & Same population & NA \\
DLP $(\mathrm{mGy}-\mathrm{cm})$ & $\mathbf{8 0 6 . 2} \pm \mathbf{4 3 7 . 7}$ & $822.1 \pm 526.1$ & 0.92 \\
\hline
\end{tabular}

Abbreviations: BMI, body mass index; DLP, dose-length product; $\mathrm{mGy}-\mathrm{cm}$, milligray-centimeter; NA, not applicable. 
Spinal computer navigation may be referenced based off of a preoperative CT scan which is then "point matched" intraoperatively to known anatomic structures utilizing a navigated instrument. An alternative approach is to perform imaging on the table during the procedure (ie, an intraoperative spin with the O-arm) and then utilize these data for guidance during the surgery. One potential advantage to intraoperative imaging includes the maintenance of relationships between various anatomic structures, which potentially may differ in the supine position during a traditional CT scan. One potential disadvantage to this approach may be longer operative times given the extra step of obtaining the spin during the surgical procedure. In our study, intraoperative spins were used in all cases as per surgeon preference.

Although the results did approach statistical significance in the main study group, the clinical significance of this must also be considered. The total difference in mean radiation DLP between the 2 groups was $125.9 \mathrm{mGy}-\mathrm{cm}$. In biologic effective dose equivalent units, the average radiation dose for $\mathrm{O}$-arm navigation would be 14.3 millisieverts ( $\mathrm{mSv}$ ), compared to $14.6 \mathrm{mSv}$ for a lumbar spine CT scan. Considering that an average pelvis $\mathrm{x}$-ray equates to $0.6 \mathrm{mSv}$, we would argue that a difference of less than that value in terms of effective radiation dose is of limited clinical significance in this context.

The results of the present study are similar to the small number of previously published results on this topic. Most recently and most relevant was a study published by Balling ${ }^{9}$ which found average DLP for 306 O-arm navigated fusions to be $865.1 \mathrm{mGy}-\mathrm{cm}$ (798.3 $\mathrm{mGy}-\mathrm{cm}$ in this study) and average DLP for 100 diagnostic lumbar CT scans to be $575.5 \mathrm{mGy}$ $\mathrm{cm}$ (924.2 $\mathrm{mGy}-\mathrm{cm}$ for this study). A notable difference between the aforementioned study and our study is our noticeably higher DLP values observed for diagnostic CT scans, while the radiation exposure for $\mathrm{O}$-arm navigation was very similar. The ultimate conclusion in the study by Balling ${ }^{9}$ was that $\mathrm{O}$-arm navigation initially resulted in more radiation exposure to the patient; however, after a learning curve of 250 cases, the average DLP for O-arm cases was $562.1 \mathrm{mGy}-\mathrm{cm}$, which was equivalent to their lumbar $\mathrm{CT}$ scans.

In a comparison of $\mathrm{O}$-arm to intraoperative mobile CT (iCT-Airo), Scarone et al found an average radiation dose for 166 patients in the $\mathrm{O}$-arm group of $19.12 \mathrm{mSv}$ (versus $14.3 \mathrm{mSv}$ in this study). ${ }^{12}$ The iCT-Airo group included 97 patients with an average exposure of $15.82 \mathrm{mSv}$. Other studies have found similar radiation exposure values for $\mathrm{O}$-arm versus CT scan using dosimeter quantification and cadaveric models. 6 ,7

While this study considered radiation exposure to the patient only, exposure of the surgical team is also an important safety concern. At our institution the surgical team briefly leaves the operating room while the $\mathrm{O}$-arm spins, meaning that there is no radiation exposure in this case. Previous studies have also documented less radiation exposure to the surgeon for pedicle screw placement using $\mathrm{O}$-arm as opposed to $\mathrm{C}$-arm. ${ }^{6}$

There are multiple limitations of this study, chief of which is that reported DLP represents an output of radiation and not a true measured dose received by various tissues within a patient. In addition to this, the control group included standard lumbar spine CT scans, and not navigation-protocol-directed scans, which could theoretically differ if they were intended to be used for calibration of intraoperative navigation. It would be of further use to study these comparisons more directly by independently measuring radiation exposure at various locations and comparing navigation-protocol CT scans.

\section{CONCLUSION}

Overall the study does provide a clinically useful estimate of radiation exposure from $\mathrm{O}$-arm navigation for lumbar spine fusion compared to standard lumbar CT scans. Given the similarity in radiation exposure between $\mathrm{O}$-arm navigation and lumbar CT scan, surgeons should rely on other factors to guide decision making in regard to mode of imaging for computer navigation. Knowledge of this comparison and total radiation exposure will also be useful for patient education and shared decision making in regard to navigated procedures.

\section{ACKNOWLEDGMENTS}

The authors would like to recognize the contributions of Erik Yannone who assisted in identification of eligible patients to be included in the study.

\section{REFERENCES}

1. Overley SC, Cho SK, Mehta AI, Arnold PM. Navigation and robotics in spinal surgery: where are we now? Neurosurgery. 2017;80(3S):S86-S99. doi:10.1093/neuros/nyw077. 
2. Shin M-H, Hur J-W, Ryu K-S, Park C-K. Prospective comparison study between the fluoroscopy-guided and navigation coupled with O-arm-guided pedicle screw placement in the thoracic and lumbosacral spines. J Spinal Disord Tech. 2015;28(6):E347-351. doi:10.1097/BSD.0b013e31829047a7.

3. Van de Kelft E, Costa F, Van der Planken D, Schils F. A prospective multicenter registry on the accuracy of pedicle screw placement in the thoracic, lumbar, and sacral levels with the use of the O-arm imaging system and StealthStation Navigation. Spine. 2012;37(25):E1580-1587. doi:10.1097/BRS. 0b013e318271b1fa.

4. Slomczykowski M, Roberto M, Schneeberger P, Ozdoba C, Vock P. Radiation dose for pedicle screw insertion. Fluoroscopic method versus computer-assisted surgery. Spine. 1999;24(10):975-982; discussion 983. doi:10.1097/00007632199905150-00009.

5. Su AW, McIntosh AL, Schueler BA, et al. How does patient radiation exposure compare with low-dose O-arm versus fluoroscopy for pedicle screw placement in idiopathic scoliosis? J Pediatr Orthop. 2017;37(3):171-177. doi:10.1097/ BPO.0000000000000608.

6. Tabaraee E, Gibson AG, Karahalios DG, Potts EA, Mobasser J-P, Burch S. Intraoperative cone beam-computed tomography with navigation (O-ARM) versus conventional fluoroscopy (C-ARM): a cadaveric study comparing accuracy, efficiency, and safety for spinal instrumentation. Spine. 2013;38(22):1953-1958. doi:10.1097/BRS.0b013e3182a51d1e

7. Lange J, Karellas A, Street J, et al. Estimating the effective radiation dose imparted to patients by intraoperative cone-beam computed tomography in thoracolumbar spinal surgery. Spine. 2013;38(5):E306-312. doi:10.1097/BRS. 0b013e318281d70b

8. Pitteloud N, Gamulin A, Barea C, Damet J, Racloz G, Sans-Merce M. Radiation exposure using the O-arm ${ }^{\circledR}$ surgical imaging system. Eur Spine J. 2017;26(3):651-657. doi:10.1007/ s00586-016-4773-0.

9. Balling $H$. Time demand and radiation dose in 3D- fluoroscopy-based navigation-assisted 3D-fluoroscopy-controlled pedicle screw instrumentations. Spine. 2018;43(9):E512E519. doi:10.1097/BRS.0000000000002422.

10. Kobayashi K, Ando K, Ito K, et al. Intraoperative radiation exposure in spinal scoliosis surgery for pediatric patients using the $\mathrm{O}$-arm ${ }^{\circledR}$ imaging system. Eur J Orthop Surg Traumatol. 2018;28(4):579-583. doi:10.1007/s00590-018-2130-1.

11. Zhang J, Weir V, Fajardo L, Lin J, Hsiung H, Ritenour ER. Dosimetric characterization of a cone-beam O-arm imaging system. J Xray Sci Technol. 2009;17(4):305-317. doi:10.3233/XST-2009-0231.

12. Scarone P, Vincenzo G, Distefano D, et al. Use of the Airo mobile intraoperative CT system versus the O-arm for transpedicular screw fixation in the thoracic and lumbar spine: a retrospective cohort study of 263 patients. J Neurosurg Spine. 2018;29(4):397-406. doi:10.3171/2018.1.SPINE17927q.

Disclosures and COI: Prior to data collection being performed, this study was reviewed and approved by the Institutional Review Board. The authors declare that there are no conflicts of interest.

Corresponding Author: Alan Reynolds, MD, 1307 Federal Street, 2nd Floor, Pittsburgh, PA 15212. Phone: (412) 359-6501; Fax: (412) 359-6265; Email: Alan.reynolds@ahn.org.

Published 28 August 2020

This manuscript is generously published free of charge by ISASS, the International Society for the Advancement of Spine Surgery. Copyright (C) 2020 ISASS. To see more or order reprints or permissions, see http://ijssurgery.com. 